Monoclonal antibodies (mAbs). Clonal antibodies recognizing a single epitope on an antigen. Generally used in reference to recombinant sources.
${ }^{1}$ Botnar Research Centre, University of Oxford, Oxford, UK.

${ }^{2}$ Eli Lilly and Company, Indianapolis, IN, USA.

${ }^{3}$ Oregon Health \& Science University, Portland,

OR, USA

${ }^{4}$ Baylor University Medical Center, Dallas, TX, USA.

${ }^{5}$ Baylor Scott \& White Research Institute, Dallas, TX, USA.

凶e-mail:peter.taylor@

kennedy.ox.ac.uk

https://doi.org/10.1038 \$41577-021-00542-x

\title{
Neutralizing monoclonal antibodies for treatment of COVID-19
}

\author{
Peter C. Taylor $\mathbb{1}^{1 凶}{ }^{凶}$, Andrew C. Adams², Matthew M. Hufford², Inmaculada de la Torre ${ }^{2}$, \\ Kevin Winthrop ${ }^{3}$ and Robert L. Gottlieb (iD) 4,5
}

Abstract | Several neutralizing monoclonal antibodies (mAbs) to severe acute respiratory syndrome coronavirus 2 (SARS-CoV-2) have been developed and are now under evaluation in clinical trials. With the US Food and Drug Administration recently granting emergency use authorizations for neutralizing mAbs in non-hospitalized patients with mild-to-moderate COVID-19, there is an urgent need to discuss the broader potential of these novel therapies and to develop strategies to deploy them effectively in clinical practice, given limited initial availability. Here, we review the precedent for passive immunization and lessons learned from using antibody therapies for viral infections such as respiratory syncytial virus, Ebola virus and SARS-CoV infections. We then focus on the deployment of convalescent plasma and neutralizing mAbs for treatment of SARS-CoV-2. We review specific clinical questions, including the rationale for stratification of patients, potential biomarkers, known risk factors and temporal considerations for optimal clinical use. To answer these questions, there is a need to understand factors such as the kinetics of viral load and its correlation with clinical outcomes, endogenous antibody responses, pharmacokinetic properties of neutralizing $m A b s$ and the potential benefit of combining antibodies to defend against emerging viral variants.

In the midst of the current COVID-19 pandemic, a variety of prophylactic and therapeutic treatments are being developed or repurposed to combat COVID-19. Monoclonal antibodies (mAbs) that can bind to and 'neutralize' the virus in infected patients are a novel class of antiviral intervention ${ }^{1,2}$. Neutralizing $\mathrm{mAbs}$ are recombinant proteins that can be derived from the $\mathrm{B}$ cells of convalescent patients or humanized mice (FIG. 1). High-throughput screening of these B cells permits the identification of antibodies with the necessary specificity and affinity to bind to a virus and block entry of the virus, therefore abrogating pathology associated with productive infection. These mAbs are termed 'neutralizing' and can ultimately be used as a type of passive immunotherapy (detailed later) to minimize virulence. In this Review, we highlight the relative value that neutralizing mAbs can provide for patients and physicians, and go on to examine the role of these agents among the spectrum of potential treatments for COVID-19.

In the United States, three anti-severe acute respiratory syndrome coronavirus 2 (SARS-CoV-2) $\mathrm{mAb}$ therapies have been granted emergency use authorization (EUA) for treatment of non-hospitalized patients with mild-to-moderate COVID-19 - these are bamlanivimab as a monotherapy, and bamlanivimab together with etesevimab or casirivimab with imdevimab as a combination therapy ${ }^{3-5}$. Therefore, several questions need to be addressed about the potential clinical use of neutralizing SARS-CoV-2 mAbs: who should get them; what is the best dose and frequency; when in the course of the infection will they be most effective; what is the duration of the protection they provide; and what is their associated benefit-to-risk ratio? In addition, neutralizing $\mathrm{mAbs}$ may have a prophylactic role in individuals deemed to be at high risk of severe COVID-19. Indeed, preliminary non-peer-reviewed preprint data suggest that $\mathrm{mAbs}$ prevent COVID-19 in high-risk individuals potentially exposed to SARS-CoV-2 in nursing homes or within households ${ }^{6,7}$.

While vaccines remain the best strategy to prevent COVID-19, mAbs could potentially benefit certain vulnerable populations before or after exposure to SARS-CoV-2, such as the unvaccinated or recently vaccinated high-risk patients. The antiviral activity seen with neutralizing $\mathrm{mAb}$ treatment emphasizes the importance of early intervention to help counter the devastating impact the virus has had in such vulnerable populations and in other high-risk patients. However, mAbs are complicated to produce and may be limited in initial supply. Furthermore, any protection offered would be temporary, and the duration of effective protection remains to be determined. Answers to these questions will allow 


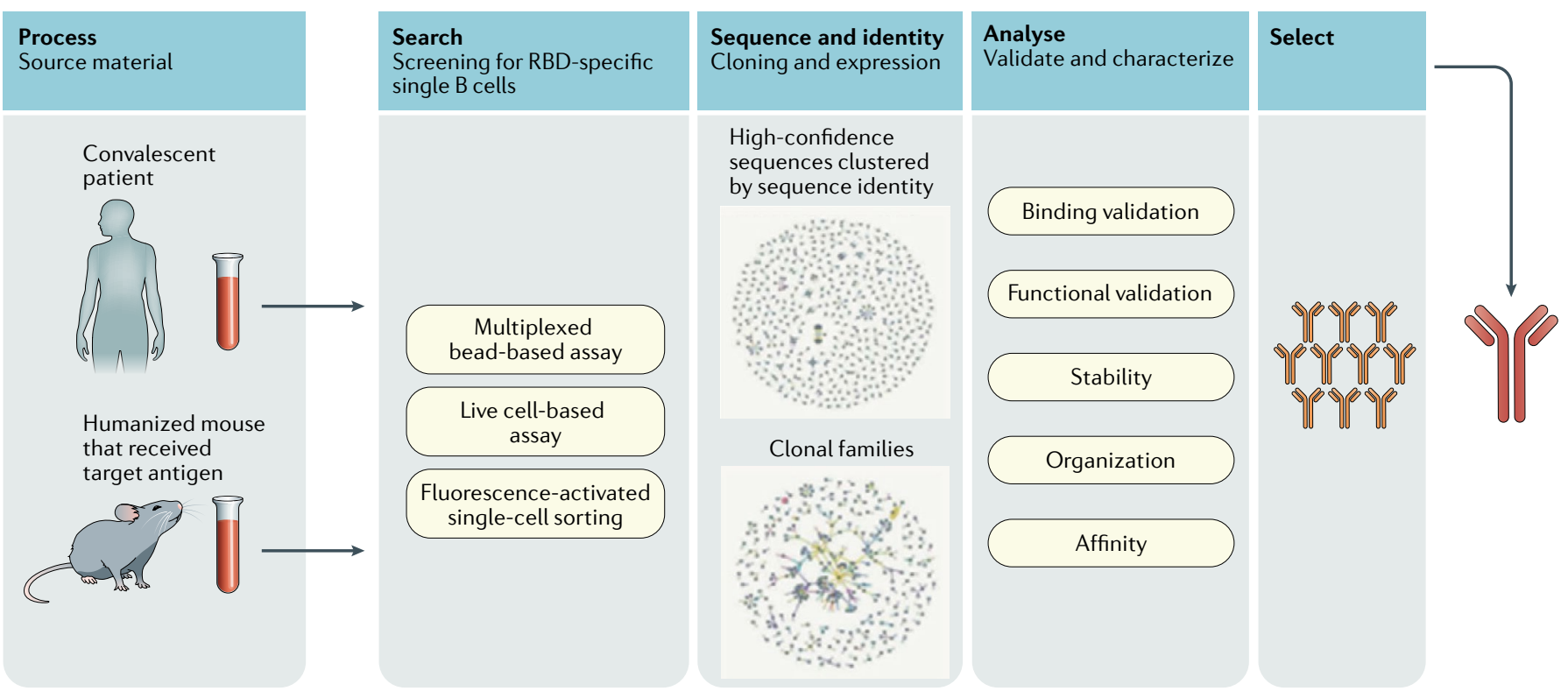

Fig. 1 | Neutralizing monoclonal antibodies: identification, selection and production. The neutralizing monoclonal antibodies (mAbs) given emergency use authorization for treatment of COVID-19 were derived from either convalescent patients or humanized mice exposed to severe acute respiratory syndrome coronavirus 2 (SARS-CoV-2) antigens. However, $\mathrm{mAbs}$ can be generated by multiple methods, including from vaccinated individuals (not depicted here). The pathways of $\mathrm{mAb}$ generation depicted here converge in the process of selection and production. RBD, receptor-binding domain.

Passive immunotherapy The introduction of monoclonal or polyclonal antibodies derived from non-human or human blood products to provide protection against infection or envenomation.

\section{Emergency use}

authorization

(EUA). A mechanism to facilitate the availability and use of medical countermeasures during a public health emergency. US Food and Drug Administration issuance of an EUA permits the use of unapproved medical products or unapproved uses of approved medical products when no adequate alternatives are available.

Convalescent plasma therapy

(CPT). The administration of donated plasma from an individual who has had an illness and recovered from it (for example, previously infected with severe acute respiratory syndrome coronavirus 2 (SARS-CoV-2) but has now recovered), to an infected individual. The recovered patient's plasma contains antibodies, which when administered to other patients is thought to boost the ability to fight disease. the most efficacious use of these novel and potentially life-saving treatments, as we discuss herein.

\section{Passive immunization}

More than 125 years ago, the first major success in modern immunological intervention was developed: a therapeutic serum from animals actively immunized against diphtheria toxin ${ }^{8,9}$. Paul Ehrlich later produced a seminal article tying the curative antiserum to neutralizing antibodies ${ }^{10}$. Today, passive immunization involves infusion of antigen-specific mAbs or polyclonal antibodies derived from non-human or human blood products. While polyclonal antibodies collected from immunized animals are the primary source of antisera, there is a risk of 'serum sickness', especially after repeated exposures, as the recipient may generate an immune response against antibodies of non-human origin. These risks are mitigated with the use of convalescent plasma from human patients. With careful screening (for example, to assess for the presence of infectious agents and to establish antibody titre and neutralizing capacity), convalescent plasma therapy (CPT) can be effective with minimal safety risks.

Before the current pandemic, CPT was used to treat infections with influenza virus ${ }^{11,12}$, respiratory syncytial virus $(\mathrm{RSV})^{13}$, Ebola virus ${ }^{14}$ and other coronaviruses ${ }^{12,15-17}$. CPT appears most efficacious when used early after the onset of symptoms, rather than during severe or prolonged infection ${ }^{12,15,18}$. It also has the potential to provide protection for the immunocompromised or unvaccinated high-risk individuals recently exposed to infection ${ }^{13,15}$. Administration of plasma with higher titres of neutralizing antibodies is associated with improved clinical outcomes ${ }^{17}$; however, the antibody titres of convalescent plasma differ considerably ${ }^{19}$. CPT can be convenient and adaptable for use in resource-poor settings ${ }^{14}$ and can be rapidly deployed to combat novel virus outbreaks.

The antipathogen antibodies from convalescent plasma can mitigate infection by two main mechanisms: namely, antibody effector activity and pathogen neutralization. However, in rare cases, pathogen-specific antibodies can augment virulence in a process termed 'antibody-dependent enhancement' (ADE) (FIG. 2). ADE can occur via two distinct mechanisms. First, pathogen-specific antibodies could increase infection by promoting virus uptake and replication in $\mathrm{Fc} \gamma$ receptor-expressing immune cells (for example, as is seen in dengue haemorrhagic virus infection of macrophages). With SARS-CoV and SARS-CoV-2 $\left(\mathrm{REF}^{20}{ }^{20}\right.$, in vitro evidence amassed to date indicate that these non-lymphotropic coronaviruses are unable to productively replicate within haematopoietic cells ${ }^{21}$. Alternatively, ADE can be mediated via increased immune activation by Fc-mediated effector functions or immune complex formation ${ }^{22}$. In the case of respiratory virus infections, the resulting immune cascade can contribute to lung disease. While the hallmarks of severe COVID-19 have features that overlap with this type of $\mathrm{ADE}$, there is currently no definitive evidence to show ADE occurs with SARS-CoV-2 infection ${ }^{22}$.

Nonetheless, steps may be considered to mitigate the potential risk of ADE. When feasible (as with neutralizing $\mathrm{mAb}$ therapy), the $\mathrm{Fc}$ region of the antibody can be modified to render it incapable of engaging effector immune responses. In the case of CPT, the potential risk of ADE can be reduced by administrating high amounts of pathogen-specific antibodies and using plasma with high-affinity neutralizing antibodies ${ }^{20}$. However, these strategies must be balanced with the 


\section{Antibody-dependent} enhancement

(ADE). The promotion of viral uptake into cells owing to the presence of suboptimal antibodies. ADE can result in enhanced viral replication and/or aberrant inflammation.

Randomized controlled trials

(RCTs). Studies that randomly assign participants into an experimental group or a control group to measure the effectiveness of a new intervention or treatment. potential loss of efficacy from effector-mediated activity. As shown in a non-primate model of SARS-CoV, neutralizing anti-receptor-binding domain (RBD) or anti-heptad repeat 2 antibodies provided protective immunity, whereas antibodies specific for other $S$ protein epitopes could trigger $\mathrm{ADE}^{23}$. Furthermore, in randomized controlled trials (RCTs), passive immunization with anti-S protein-neutralizing $\mathrm{mAbs}$ did not provide clinical evidence of ADE in non-hospitalized patients with COVID-19 (REFS $3,24,25)$.

The shortage of large RCTs of CPT has limited our understanding of the relative benefit-to-risk profile of

a
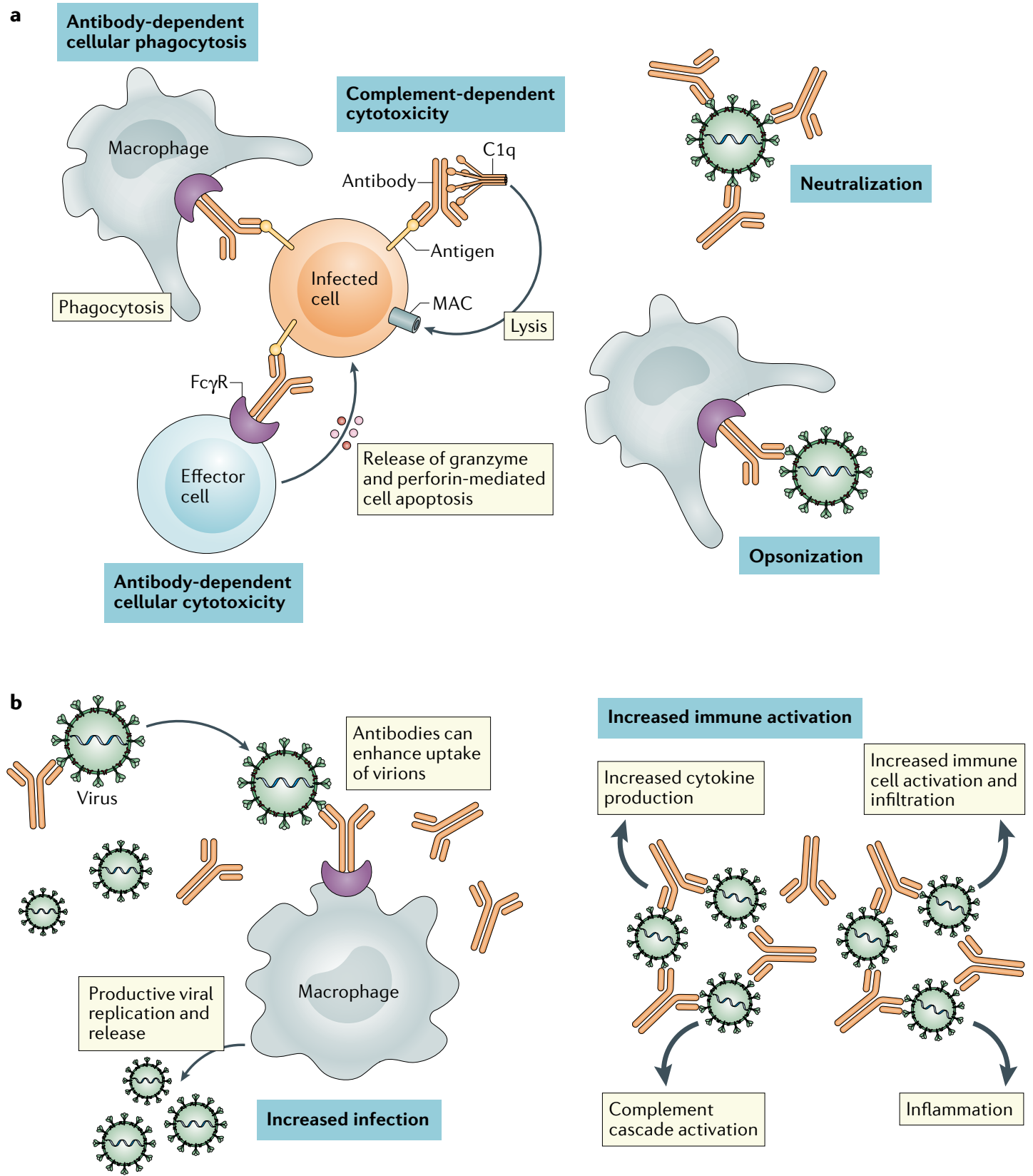

Fig. 2 | Mechanism of action of monoclonal antibodies for viral infection and antibody-dependent enhancement. a Monoclonal antibodies can directly interfere with viral pathogenesis in multiple ways. First, binding of a neutralizing antibody to the virion can prevent target cell binding and/or fusion. Furthermore, antibody binding opsonizes the virions or infected cells for phagocytic uptake. If viral proteins are intercalated into target cell membranes during viral egress, monoclonal antibodies can facilitate target cell death via complement fixation and membrane attack complex (MAC) activation or antibody-dependent cytotoxicity. These mechanisms may result in apoptosis or necrosis of the infected cell. b | In some instances, opsonization of a virion can facilitate viral pathogenesis in a process termed 'antibody-dependent enhancement' (ADE). ADE can occur via two distinct mechanisms. First, pathogen-specific antibodies could increase infection via viral uptake and replication in $\mathrm{Fc} \gamma$ receptor $(\mathrm{Fc} \gamma \mathrm{R})$-expressing immune cells. Secondly, ADE can be mediated via increased immune activation by Fc-mediated effector functions or immune complex formation. The process of ADE and its potential impact during severe acute respiratory syndrome coronavirus 2 (SARS-CoV-2) infection is expertly reviewed by Lee et al. ${ }^{22}$. 
this treatment option. Furthermore, logistical difficulties can complicate the application of CPT. According to the EUA from the US Food and Drug Administration for CPT in patients with COVID-19, convalescent patients should be symptom-free for a minimum of 2 weeks and have high titres of anti-SARS-CoV-2 antibodies; low-titre donations could be used for therapy following careful consideration by the health-care provider $^{26,27}$. Thus, widespread use of CPT is dependent on a readily available pool of recovering patients with high antibody titres who are willing to donate plasma, on sufficient local facilities to ensure adequate processing, screening and administration of the therapy, and on governmental coordination to regulate effective implementation.

\section{Advantages of monoclonal antibodies}

There is an increasing focus on replacing CPT with neutralizing mAbs, where dosing to ensure appropriate neutralizing capacity of the antibodies can be more precise. Today, the process to mass-produce recombinant $\mathrm{mAbs}$ has become scalable to meet demand and is cost-competitive with other treatments. Neutralizing $\mathrm{mAbs}$ overcome limitations intrinsic to CPT (for example, the risk of blood-borne diseases, time to development of detectable high-affinity antibodies and risk of low antibody titres, as well as variable epitope specificity ${ }^{28}$ ). Furthermore, a high titre of neutralizing antibodies - which current evidence indicates is necessary for the efficacy of CPT - is inherent with neutralizing mAbs. As of March 2021, at least 20 neutralizing $\mathrm{mAb}$ therapies were being tested in late-stage clinical trials or had already been approved for use in nine infectious diseases, including RSV infection and Ebola $^{29,30}$ (ClinicalTrials.gov).

Palivizumab, a neutralizing $\mathrm{mAb}$ to the fusion protein of RSV, was initially approved in 1998 as a prophylaxis for severe RSV infection in high-risk infants ${ }^{31-33}$. Previously, the standard of care for prophylaxis in these patients was monthly infusions of RSV immune globulin ${ }^{13,31}$. When administered via monthly intramuscular injections, palivizumab reduced the frequency of hospitalization and severity of RSV disease relative to placebo and was well tolerated ${ }^{31,32}$. However, palivizumab was not demonstrated in RCTs to improve clinically meaningful outcomes in infants with severe RSV infection in advanced disease stages ${ }^{34-36}$. Furthermore, monthly administration is required to maintain detectable levels of neutralizing mAbs, and as many as five doses may be needed to prevent severe or deadly infection ${ }^{37}$. A newer medication with a longer half-life (MEDI8897) is currently in phase II/III trials ${ }^{33}$.

During the Ebola virus disease outbreak in the Democratic Republic of the Congo in 2018, an openlabel RCT (PALM) investigated four intravenously administered treatments in 681 patients actively infected with Ebola virus: the antiviral remdesivir, the triple $\mathrm{mAb}$ cocktail ZMapp, the single mAb MAb114, and the triple $\mathrm{mAb}$ combination REGN-EB3 $\left(\mathrm{REF}^{38}\right)$. After an interim analysis, the first two treatments were discontinued as MAb114 monotherapy and REGN-EB3 were superior with respect to the primary outcome, patient mortality ${ }^{38}$. One potential factor the PALM study team proposed to explain the distinction between the therapeutics was that the full treatments for MAb114 and REGN-EB3 were administered as a single dose, thereby facilitating a rapid response, while ZMapp was given as three infusions. Indeed, patients treated with MAb114 and REGN-EB3 had faster rates of viral clearance, lending credence to this hypothesis. Overall, survival was higher in those who were treated early during symptom onset and had lower baseline viral loads. The relatively low efficacy of the ZMapp triple cocktail also serves as a reminder that the number of $\mathrm{mAbs}$ is not necessarily a predictor of efficacy per se, and that specific epitopes may also matter.

Two main uncertainties persist with passive immunization, spanning both neutralizing mAbs and CPT. First, does their use as a prophylactic or treatment potentially affect natural long-term immunity? Considering the large doses used and the relative half-life of antibodies ( $\sim 3$ weeks for IgG molecules), there is a pertinent consideration whether the presence of circulating neutralizing mAbs could impact active immunity, whether through memory from infection or vaccination. In RSV infection, rodent and primate infection models indicate that the passive transfer of antibodies does diminish the development of humoral immunity in the recipient; however, long-term memory was sufficient to protect the hosts from reinfection, largely owing to an intact $\mathrm{T}$ cell memory compartment $^{39,40}$. Considering the limitations in translating data from animal models (where RSV replication is attenuated relative to that in its human host), additional data, particularly from clinical trials, will provide critical insight with regard to this potential challenge.

Second, could resistant viral variants emerge that limit the effectiveness of the therapies? The polyclonal nature of CPT, in which a spectrum of differentiated antibodies target multiple epitopes of the pathogen, may help to reduce this risk. Nevertheless, emerging preclinical data suggest SARS-CoV-2 spike (S) protein mutations escape from polyclonal serum ${ }^{41}$, and convalescent plasma has reduced neutralizing activity against some viral variants $\mathrm{s}^{42}$. For $\mathrm{mAbs}$, however, depending on the infectious agent and the epitope targeted, combinations of mAbs may be necessary to maintain efficacy and prevent treatment failure. Experience with $\mathrm{mAbs}$ targeting human immunodeficiency virus (HIV), which has a very high mutation rate, suggests that it may be more effective and durable to use multiple neutralizing antibodies (that is, combinational mAb therapy) rather than a single one $\mathrm{e}^{43-46}$. These particular mAbs to HIV also need to be broadly neutralizing and target epitopes generally conserved among viral variants. On the other hand, infections involving pathogens with lower mutation rates and/or accessible broadly conserved epitopes may not require combinational $\mathrm{mAb}$ therapy; for example, MAb114 monotherapy, which targets a broadly conserved epitope on the Ebola virus's RBD, was as effective as the combination therapy REGN-EB3 $\left(\right.$ REF. $^{38}$ ) and more effective than the ZMapp triple cocktail. It is important to note though that the global nature of the COVID-19 pandemic presents a larger risk of escape variants emerging than during the Ebola outbreak, owing to the sheer number of infections and high levels of circulating virus among populations. 
From the collective clinical data with MAb114, REGN-EB3 and palivizumab, the general benefits and risks associated with neutralizing $\mathrm{mAbs}$ are similar to those observed with traditional passive immunization against infectious agents. The agents themselves are relatively tolerable for patients, efficacious during the early onset of disease symptoms and in certain cases as a prophylactic, but with limited efficacy once infections are severe. The distinctions between these therapies are largely logistical; CPT is more rapidly implemented during an emerging pandemic when few therapeutic options are yet available, while neutralizing mAbs take time to discover and it takes time for regulatory approval for their use to be obtained as well as to scale up manufacturing capacity. The use and promise of passive immunization during the coronavirus outbreaks of the twenty-first century (that is, with SARS-CoV, Middle East respiratory syndrome-related coronavirus and SARS-CoV-2) have re-emphasized these past lessons while also highlighting additional insights, as we discuss next.

\section{Passive immunization for coronaviruses}

During the SARS epidemic in 2003, immune system kinetics following SARS-CoV infection were different in patients who recovered compared with those who finally succumbed to the viral infection and sequelae. In patients with fatal outcomes, the levels of endogenous neutralizing antibodies peaked at 15 days from symptom onset and then decreased drastically until the time of death. By contrast, in patients who went on to recover, peak neutralizing antibody responses were observed at 20 days from symptom onset ${ }^{47,48}$. Those who recovered tended to develop antibody responses with diverse isotypes (IgM, IgG and IgA) against two proteins on the virus, the nucleocapsid ( $\mathrm{N}$ ) protein and the $\mathrm{S}$ protein, while patients with fatal outcomes had restricted antibody responses to the $\mathrm{N}$ protein only. In a serological survey of confirmed convalescent serum samples, $88 \%$ had anti-SARS-CoV antibodies 31-180 days after the onset of symptoms; the geometric mean of the neutralizing antibody titre was 1:61 (REF. ${ }^{19}$ ).

Owing to the brief duration of the SARS epidemic in 2003, few observational trials examining CPT were conducted. The largest involved 80 patients and was conducted at the Prince of Wales Hospital in Hong Kong $^{15}$. Those given CPT before day 14 following onset of symptoms had a better outcome than those given CPT after day $14(P<0.001)$; mortality was also lower in the former group (6.3\% versus $21.9 \%)$. Patients who tested positive for SARS-CoV by PCR had better outcomes if they were seronegative when given $\mathrm{CPT}$ than those who were already seropositive $(66.7 \%$ versus $20 \%$; $P<0.001$ ). A summary of eight observational studies using CPT during the SARS 2003 outbreak (including the aforementioned Prince of Wales Hospital study) suggested CPT was associated with reduced mortality, shorter hospital stays and reduced overall viral loads in the respiratory $\operatorname{tract}^{12}$. The treatments were considered tolerable ${ }^{12}$; however, information on minor complications may have been under-reported. Importantly, robust studies on the effectiveness and safety of CPT were not completed, and thus these results must be interpreted with caution, particularly as patients treated at later times with CPT may demonstrate selection bias for an already refractory pathophysiology.

In SARS-CoV-2 infection, plasma collected from 175 patients who had recovered from mild COVID-19 demonstrated neutralizing antibody and S-binding antibody titres that correlated with increased age, greater inflammation (that is, higher C-reactive protein levels) and lower lymphocyte counts; the vast majority of the SARS-CoV-2 neutralizing antibodies were not cross-reactive with SARS-CoV ${ }^{49}$. Several reports indicate convalescent patients can maintain high titres of neutralizing antibodies several weeks after infection ${ }^{49-51}$.

Observational studies have reported that CPT has been associated with improved outcomes in COVID-19 $\left(\right.$ REF $\left.^{52}\right)$. For example, in a small case series in China, five critically ill patients with acute respiratory distress syndrome showed improved clinical status following CPT. Thirty-seven days after transfusion, three patients had been discharged and two were in a stable condition ${ }^{53}$. In a cohort analysis of 35,222 patients hospitalized with COVID-19 from the United States Convalescent Plasma Expanded Access Program, reduced mortality was associated with earlier time to transfusion (after diagnosis) and convalescent plasma with higher antibody levels ${ }^{18}$. Because these studies were observational, there was limited procedural control including standardization of the level of neutralizing antibodies.

In an open-label RCT of CPT for patients $(n=103)$ with severe or life-threatening COVID-19 in China, donors were required to have high levels of antibodies specific to the RBD of the S protein ${ }^{54}$. However, the study was terminated early; the hazard ratio for the time to clinical improvement within 28 days in the CPT group versus the standard treatment control group was 1.4 (favouring CPT) but was not statistically significant. The proportion of patients with severe disease who achieved the primary end point was significantly higher in the CPT group (21 of 23 patients) versus the standard treatment group ( 15 of 22 patients; $P=0.03$ ), but no distinction was noted in patients with life-threatening COVID-19 (REF. ${ }^{53}$ ). In a blinded RCT in Argentina, CPT (with a median titre of 1:3,200 anti-SARS-CoV-2 antibodies) also failed to demonstrate benefit in patients with COVID-19-associated severe pneumonia ${ }^{55}$. Furthermore, in an open-label RCT in India (PLACID) in hospitalized patients with hypoxaemia (generally comparable with the definition of severe COVID-19 from other trials), CPT did not demonstrate benefit in terms of patient mortality or transition to worsening disease ${ }^{56}$. Similarly, preliminary data from the RECOVERY RCT among 10,406 hospitalized patients showed no proof of mortality benefit in the primary end point of 28-day mortality in the CPT group versus the standard treatment group ${ }^{57,58}$.

Despite inconsistent clinical efficacy, there is evidence that CPT was associated with greater viral clearance than standard-of-care treatment ${ }^{54,56}$; collectively, this indicates increased viral clearance alone was not sufficient to clearly improve the clinical outcomes in patients with established COVID-19 (that is, in patients 
Time-weighted average An average that takes both the numerical level and the time of a particular variable into consideration

Medically attended visits Medical visits such as telemedicine visits, in-person outpatient visits to or from a medical provider, urgent care or emergency department visits, or hospitalization. hospitalized with COVID-19). Because the currently available data on CPT are derived predominantly from inpatient (severe or critical) COVID-19 RCTs, the suitability of CPT as prophylaxis or treatment at the onset of COVID-19 symptoms remains to be determined by appropriately controlled clinical trials.

\section{Monoclonal antibodies for COVID-19}

The primary antigenic epitope on SARS-CoV and SARS-CoV-2 is the S protein, which facilitates target cell binding and fusion upon engaging the cell-surface angiotensin-converting enzyme 2 (ACE2) receptor, which is found on cells in the respiratory system, gastrointestinal tract and endothelium ${ }^{59-63}$. Thus, antibodies directed
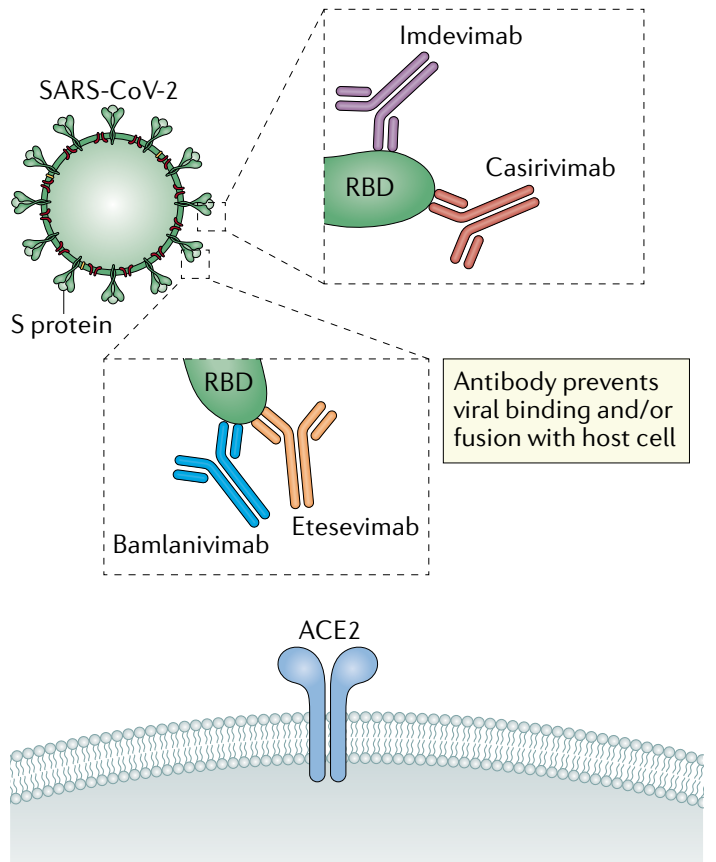

Fig. 3 | Inhibition of SARS-CoV-2 target cell engagement by neutralizing monoclonal antibodies. Neutralizing monoclonal antibodies (mAbs) being developed to combat COVID-19 are generated against the receptor-binding domain (RBD) of the spike (S) protein of severe acute respiratory syndrome coronavirus 2 (SARS-CoV-2). The anti-RBD mAbs prevent binding of the $S$ protein to its cognate receptor, angiotensin-converting enzyme 2 (ACE2), on target host cells. Three neutralizing mAb regimens have been given emergency use authorization for treatment of COVID-19. (1) Casirivimab and imdevimab bind distinct epitopes on the RBD with dissociation constants $K_{D}$ of 46 and $47 \mathrm{pM}$, respectively. Imdevimab binds the $\mathrm{S}$ protein RBD from the front or lower-left side, while casirivimab targets the spike-like loop from the top direction (overlapping with the ACE2-binding site ${ }^{3,68}$ ). (2) Bamlanivimab binds an epitope on the RBD in both its open confirmation and its closed confirmation with dissociation constant $K_{\mathrm{D}}=71 \mathrm{pM}$, covering 7 of the approximately 25 side chains observed to form contact with ACE2 (REF.). (3) Bamlanivimab and etesevimab bind to distinct, but overlapping, epitopes within the RBD of the $\mathrm{S}$ protein of SARS-CoV-2. Etesevimab binds the up/active conformation of the RBD with dissociation constant $K_{\mathrm{D}}=6.45 \mathrm{nM}\left(\mathrm{REF}^{5}{ }^{5}\right)$; it contains the LALA mutation in the Fc region, resulting in null effector function. to the $\mathrm{S}$ protein can neutralize the ability of the virus to bind and fuse with the target host cell. Humanized murine technology or convalescent plasma from recovered patients has been used to derive neutralizing mAbs targeted to the RBD of the $S$ protein ${ }^{64-66}$ (FIG. 3). To date, most advanced research efforts for therapeutic use of neutralizing $\mathrm{mAbs}$ are focusing on a handful of products in clinical development, some of which are already authorized on the basis of phase I/II and phase II data for emergency use (TABLE 1).

REGN-COV2 therapy. REGN-COV2 is a combination of two potent neutralizing mAbs - namely, casirivimab and imdevimab, which are IgG1 mAbs with unmodified $\mathrm{Fc}$ regions. These two $\mathrm{mAbs}$ were chosen from a pool of more than 200 neutralizing mAbs present in the initial isolation of thousands of antibodies and were derived from parallel efforts using humanized mice and the sera of patients recovering from COVID-19 (REFS ${ }^{67,68}$ ). The antibodies bind two distinct and non-overlapping sites on the $\mathrm{RBD}^{3,67}$. The rationale for this antibody combination is that it is unlikely that a mutation in the $\mathrm{S}$ protein of SAR-CoV-2 will simultaneously render both antibodies ineffective. In extensive in vitro testing, this combination retained its ability to neutralize all known S protein mutations ${ }^{67}$. Further, casirivimab and imdevimab combination therapy initiated antibodymediated cytotoxicity and cellular phagocytosis in virally infected cells in vitro ${ }^{3}$. This product was tested in rhesus macaques and golden hamsters infected with SARS-CoV-2, which serve as models for mild and severe disease, respectively ${ }^{69}$. In both models, prophylactic and therapeutic treatment with casirivimab and imdevimab not only resulted in a reduction in viral load but also diminished the incidence and severity of lung disease relative to a placebo.

An ongoing phase I/II/III placebo-controlled trial (NCT04425629) is investigating the safety and efficacy of a single infusion of casirivimab and imdevimab $2,400 \mathrm{mg}$ ( $n=266$, interim), 8,000 $\mathrm{mg}(n=267$, interim) or matching placebo $(n=266)$ - for symptomatic adults who have not previously been hospitalized within 3 days of a positive active SARS-COV-2 diagnosis (and within 7 days of the first symptoms) ${ }^{3}$. In the modified full analysis set for the phase I/II analysis, the median age was 42 years ( $7 \%$ aged 65 years or older), $85 \%$ of patients were white, $9 \%$ were Black and $34 \%$ were considered at high risk (for example, they were elderly, had obesity or had underlying chronic medical conditions). Pooled treatment achieved the primary end point of timeweighted average change from the baseline in viral load ( $\log _{10}$ copies per millilitre), collected from a nasopharyngeal swab, in patients with a positive baseline for viral RNA $(n=665)$. The difference in time-weighted average from day 1 through day 7 for the pooled doses of casirivimab and imdevimab compared with placebo was -0.36 $\log _{10}$ copies per millilitre $(P<0.0001)$. The combination was reported to reduce viral load particularly in patients with higher viral loads who were seronegative at the baseline $e^{3,70}$. On a key clinical end point, a lower proportion of patients treated with casirivimab and imdevimab had COVID-19-related medically attended visits 
Table 1 | Neutralizing monoclonal antibodies for SARS-CoV-2 currently in development up to 11 December 2020

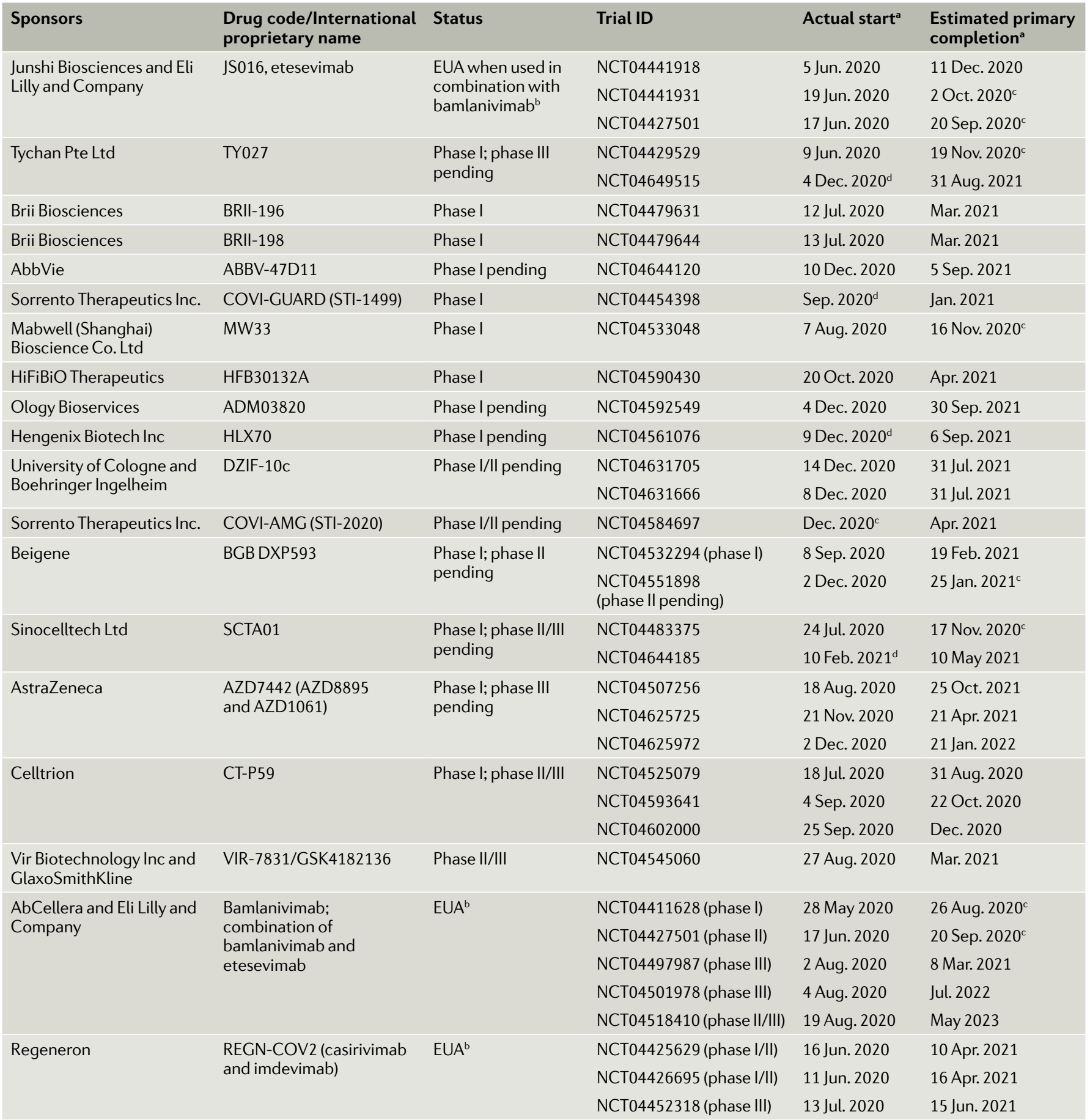

A complete list can be found at COVID-19 Biologics Tracker. EUA, emergency use authorization; SARS-CoV-2, severe acute respiratory syndrome coronavirus 2.

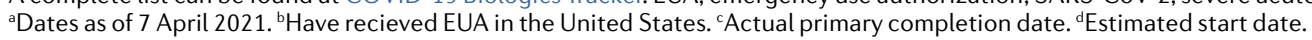

( $2.8 \%$ for pooled doses versus $6.5 \%$ for placebo). In post hoc analyses, a lower proportion of patients treated with casirivimab and imdevimab had COVID-19-related hospitalizations or emergency department visits compared with patients who received placebo $(2 \%$ versus $4 \%)$. The absolute risk reduction for casirivimab and imdevimab compared with placebo was greater for patients at high risk of progression to severe COVID-19 and/or hospitalization (3\% versus $9 \%$ ). Collectively, these results supported the EUA of Regeneron's casirivimab and imdevimab cocktail in the United States in November $2020\left(\right.$ REF $\left.^{71}\right)$.

Bamlanivimab monotherapy. Bamlanivimab is a potent neutralizing $\mathrm{mAb}$ (IgG1 with an unmodified Fc region) to the $\mathrm{S}$ protein that was derived from the convalescent plasma of a patient who had COVID-19 (REFS $\left.{ }^{24,66}\right)$. Bamlanivimab binds the $S$ protein's $\mathrm{RBD}$, engaging its cognate epitope in both up and down conformations, which makes this antibody potentially useful as a 
monotherapy. There have been historical precedents for the effectiveness of neutralizing mAbs as a monotherapy (for example, MAb114 for Ebola) ${ }^{38}$. To assess theoretical risk of ADE, bamlanivimab was studied in primary human macrophages and immune cell lines exposed to SARS-CoV-2 at concentrations down to 100 -fold below the effective concentration for half-maximum response, and in these studies, it did not demonstrate productive viral infection ${ }^{4}$. Prophylactic efficacy was tested in rhesus macaques given bamlanivimab 24 hours before a virus challenge ${ }^{66}$. The symptoms in this model were mild overall, but the treatment significantly decreased viral load and replication in the respiratory tract following inoculation, supporting its antiviral efficacy.

In the phase II portion of the ongoing phase II/III BLAZE-1 trial (NCT04427501), ambulatory adults with mild-to-moderate symptoms of COVID-19 within 3 days of a first-positive nasopharyngeal swab positive for SARS-CoV-2 received a single infusion of one of three doses of bamlanivimab (700, 2,800 or 7,000 mg) or placebo in an outpatient setting ${ }^{24}$. A pre-planned interim analysis was conducted of 452 patients who had reached day 11 following infusion (median age $45-46$ years ( $12 \%$ aged 65 years or older), $88 \%$ white, $6 \%$ Black and $68 \%$ at high risk (for example, they were elderly, had obesity or had underlying chronic medical conditions) $)^{24}$. The study revealed the viral clearance time course via the intrinsic immune response and the enhanced clearance with neutralizing $\mathrm{mAb}$ infusion, concomitant with improved clinical response. Viral loads were assayed from serial nasopharyngeal swabs, with postinfusion measurements enabled by a novel partnership with home-health research. Following infusion, the log viral load had begun decreasing relative to the baseline as early as the first postinfusion assessment on day 3 ( -0.85 for placebo versus -1.35 for pooled bamlanivimab doses), continued to decrease on day 7 ( -2.56 for placebo versus -2.90 for pooled bamlanivimab doses) and further decreased by day 11 ( -3.47 for placebo versus -3.70 for pooled bamlanivimab doses). In a post hoc analysis, patients with early persistent high viral load (described as log viral load of 5.27 or greater at trial day 7) had a higher risk of hospitalization, and the risk was further increased for elderly patients and patients with obesity. Clinical evidence demonstrating the efficacy of bamlanivimab came from two predefined secondary end points. First, at day 29, the percentage of patients who were hospitalized with COVID-19 was $6.3 \%$ for the placebo group and reduced to $1.6 \%$ for the group with pooled bamlanivimab doses. In post hoc analyses, hospitalization among elderly patients ( 65 years or older) or patients with obesity (body mass index $35 \mathrm{~kg} \mathrm{~m}^{-2}$ or greater) was $15 \%$ for the placebo group and $4 \%$ for the group who received pooled bamlanivimab doses. Absolute risk reduction for hospitalizations was more evident for patients with risk factors. Second, amelioration of baseline symptoms was greater for the pooled bamlanivimab doses than for placebo from day 2 through to day 11 . Collectively, these results supported the EUA of bamlanivimab monotherapy in the United States and Canada in November 2020 (REF. ${ }^{4}$.
Bamlanivimab and etesevimab. Other treatment arms of the BLAZE-1 trial studied bamlanivimab together with etesevimab (an S protein-binding IgG1 with a modified Fc region, resulting in null effector function $)^{25,72}$. Bamlanivimab and etesevimab together significantly decreased viral load (mean changes from the baseline and percentage of patients with persistent high viral load) compared with placebo at day 3 to day 11 (REF. ${ }^{25}$ ). Bamlanivimab- and etesevimab-treated patients had fewer COVID-19-related hospitalizations relative to the placebo group (5.8\% for placebo reduced to $0.9 \%$ for bamlanivimab together with etesevimab). Recently released placebo-controlled phase III data from 1,035 patients randomized 1:1 to receive bamlanivimab together with etesevimab versus placebo demonstrated that in high-risk ambulatory patients (including patients aged 12-17 years with specific risk factors and patients aged 18 years or older with specific adult risk factors) treatment with bamlanivimab and etesevimab together was associated with a $70 \%$ reduction in COVID-19-related hospitalizations and deaths relative to placebo treatment (7.0\% for placebo reduced to $2.1 \%$ for bamlanivimab together with etesevimab $)^{25,73}$. On the basis of these data, an additional EUA of bamlanivimab together with etesevimab has been issued ${ }^{5}$.

Monoclonal antibody therapies in severe COVID-19. There are concurrent studies investigating neutralizing $\mathrm{mAbs}$ for patients hospitalized with severe COVID-19. The REGN-COV2 trial in hospitalized patients enrols patients with or without supplemental oxygen and is ongoing ${ }^{74,75}$. In prospectively designed analysis of REGN-COV2, there may be clinical benefit in patients treated with casirivimab and imdevimab and who were seronegative at the time of treatment ${ }^{76}$. In the ACTIV-3 $\operatorname{RCT}(n=326,1: 1$ randomization), bamlanivimab added to standard of care (typically including remdesivir) did not demonstrate additional clinical benefit in hospitalized patients ${ }^{77}$. In line with similar studies investigating CPT or neutralizing mAbs for patients with severe viral disease (including COVID-19) ${ }^{15,18,34-36,54,56}$, the evidence indicates that rapid viral clearance, in itself, is insufficient. Rather, additional factors, such as an excessive immune response, are the primary drivers for continued disease in this particular patient population. Thus, early disease seen in outpatients is likely virally driven, whereas the pathophysiology for inpatient advanced disease is predominantly a postviral or periviral phenomenon, with clinical status uncoupled from viral load.

\section{Adverse events associated with monoclonal antibody} therapies. In terms of risk associated with $\mathrm{mAb}$ treatment of COVID-19, treatment-associated adverse events were comparable to those with placebo. The most frequent side effects observed in RCTs include nausea, diarrhoea, dizziness, headache and vomiting ${ }^{24,25,78}$. One per cent of patients receiving casirivimab and imdevimab reported a grade 2 or higher infusion-related reaction within 4 days of administration (comparable to $1 \%$ reported for placebo treatment $)^{78}$. In the phase II portion of BLAZE-1, nine patients reported an infusion-related reaction 
(1.9\% (6/309) with bamlanivimab monotherapy, $1.8 \%$ (2/112) with bamlanivimab and etesevimab together, and $0.6 \%(1 / 156)$ with placebo). Most reactions occurred during infusion; these were mild in severity and were not dose related ${ }^{25}$. Regarding evidence of ADE, in vitro data indicate neutralizing $\mathrm{mAbs}$ do not enhance productive infection of immune cells with SARS-CoV-2 (REFS $\left.{ }^{3,4}\right)$. From the clinical data available to date, there is no clear evidence these therapies result in enhanced immune responses consistent with $\mathrm{ADE}^{24,25,78}$. Furthermore, the safety profiles of modified and modified plus unmodified mAbs to treat SARS-CoV-2 infection are similar, suggesting that ADE may not play a role in clinical outcomes ${ }^{25}$.

Emergence of drug-resistant SARS-CoV-2 strains. For patients with COVID-19 who receive neutralizing $\mathrm{mAbs}$, there is potential for the development of drug-resistant variants, which become more obvious when selective pressure is applied in the setting of drug treatment ${ }^{78,79}$. For bamlanivimab, non-clinical studies using serial passage of SARS-CoV-2 and directed evolution of the SARS-CoV-2 S protein identified viral variants (E484D/K/Q, F490S, Q493R and S494P, amino acid substitutions in the $\mathrm{S}$ protein $\mathrm{RBD}$ ) that had increased resistance to this drug ${ }^{4}$.

In clinical trials of bamlanivimab, genotypic and phenotypic testing are monitoring SARS-CoV-2 strains for potential $S$ protein variations that are associated with bamlanivimab resistance. In clinical trials of bamlanivimab, viral sequencing is being performed for all patients, regardless of treatment status/progression. In other studies where only treatment failures are sampled, the selective pressure exerted by the antiviral activity cannot be assessed. In the BLAZE-1 RCT, which was limited to US investigative sites, known bamlanivimab-resistant variants at the baseline were observed at a frequency of $0.27 \%$ to date ${ }^{4}$. In the same trial, treatment-emergent variants were detected at $\mathrm{S}$ protein amino acid positions E484, F490 and S494 (including E484A/D/G/K/Q/V, F490L/S/V and S494L/P); considering all variants at these positions, $9.2 \%$ and $6.1 \%$ of participants in the 700-mg bamlanivimab arm (the EUA dose) harboured such a variant after the baseline at allele fractions of $15 \%$ or greater and $50 \%$ or greater, respectively, compared with $8.2 \%$ and $4.1 \%$, respectively, of participants in the placebo arm. Most of these variants were first detected on day 7 following infusion, and were detected at only a single time point. The clinical impact of these variants is currently unknown ${ }^{4}$.

As with bamlanivimab, casirivimab and imdevimab therapy has the potential to lead to the development of resistant viral variants. In non-clinical studies, serial passage of vesicular stomatitis virus (VSV) encoding the SARS-CoV-2 S protein in the presence of the drugs identified escape variants with reduced susceptibility to casirivimab (K417E/N/R, Y453F, L455F, E484K, F486V and Q493K) or imdevimab (K444N/Q/T and V445A) ${ }^{3}$. Each viral variant showing reduced susceptibility to one $\mathrm{mAb}$ remained susceptible to the other $\mathrm{mAb}$; all identified variants retained susceptibility to the combination. In a separate experiment, neutralization assays were performed with VSV pseudotyped with 39 variants of the $\mathrm{S}$ protein identified in circulating SARS-CoV-2. The G476S, S494P and Q409E variants had reduced susceptibility (5-fold, 5-fold, and 4-fold, respectively) to casirivimab, and the $\mathrm{N} 439 \mathrm{~K}$ variant had reduced susceptibility (463-fold) to imdevimab. The casirivimab and imdevimab combination was active against all individual variants tested ${ }^{3}$. It has been reported that the combination of mutants at residues 417 and 439 may abrogate the effectiveness of the casirivimab and imdevimab combination $^{80}$.

In the casirivimab and imdevimab RCT NCT04425629, interim data indicated only one variant (G446V) detected in $4.5 \%$ of participants at an allele fraction of $15 \%$ or greater, each detected at a single time point ${ }^{3}$. The clinical impact is unknown. In a VSV pseudoparticle neutralization assay, the G446V variant had reduced susceptibility to imdevimab (135-fold) but retained susceptibility to both casirivimab alone and the casirivimab and imdevimab combination.

However, not all variants must be considered clinically relevant mutations associated with resistance to treatment. During the Ebola outbreak in 2018, a genomic assessment of 48 viral genomes determined that this outbreak was due to a distinct viral variant. The sequence information allowed researchers to evaluate the relevance of the distinct mutations to the available vaccine and therapeutics and to conclude that the neutralizing antibodies MAb114 and ZMapp would likely be effective against the currently circulating variant ${ }^{81}$. A similar practice for SARS-CoV-2 surveillance may be prudent to determine whether emergent $S$ protein variants pose a threat to the efficacy of neutralizing $\mathrm{mAb}$ therapies.

Indeed, three SARS-CoV-2 variants of particular interest have been identified and are circulating globally. In the United Kingdom, a variant called 'B.1.1.7' with a large number of mutations was identified in the autumn of 2020. In South Africa, a variant called 'B.1.351 was identified. Originally detected in early October 2020, B.1.351 shares some mutations with B.1.1.7. In Brazil, a variant called 'P.1' was identified that contains a set of additional mutations that may affect its ability to be recognized by first-generation neutralizing $\mathrm{mAbs}$ and by the immune responses generated by first-generation vaccines. Although these variants have been detected in the United States, according to real-time data accessed via the GISAID COVID-19 variant tracker ${ }^{82}$ these COVID-19 variants do not currently represent a significant proportion of COVID-19 infections in the United States $^{82,83}$, while recent California (B.1.427/B.1.429) and New York (B.1.526) variants do. To date, the effect of these variants on the neutralizing capacity of vaccines and $m A b s$ is unknown. A recent preprint suggests that the variants identified in the United Kingdom and South Africa are more resistant to CPT and vaccine sera ${ }^{42}$.

Bamlanivimab and imdevimab maintain full neutralization activity against the primary SARS-CoV-2 receptor-binding site variants (69-70del and N501Y) implicated in the strain originating in the United Kingdom, suggesting that these mAbs should maintain full activity against the new strain originating in the United Kingdom ${ }^{42,84}$. From what is known about the 
strains that were first identified in South Africa, Brazil as well as the ones in California and New York, it appears that some of the first generation of antibody therapies may not be as effective and it will be important for physicans to refer to the most up to date factsheet ${ }^{3-5,42}$.

\section{Clinical use in COVID-19}

Bamlanivimab, bamlanivimab together with etesevimab, and casirivimab with imdevimab decrease viral load when given early on in the course of SARS-CoV-2 infection and favourably impact clinical outcomes for patients with mild-to-moderate COVID-19 $\left(\mathrm{REFS}^{24,70}\right)$. Although full clinical trial data are pending, top-line and interim results from multiple trials suggest that the therapies may also function as prophylaxis in at-risk patients recently exposed to SARS-CoV-2 $\left(\mathrm{REFS}^{6,7}\right)$. One signal emerging from early data is that patients with persistently higher viral loads progress more frequently towards medically attended visits, emergency department visits or hospitalization, and this effect is most pronounced for patients with pre-existing risk factors for disease progression ${ }^{3,24}$ It remains a tenet that antivirals, whether small molecules or neutralizing mAbs, work best when deployed early. By extrapolation from early viral load data, ideally patients would receive treatment as soon as possible (that is, within hours to days following a positive test or symptom onset). In the trial setting, by day 7 to day 11 most patients either are progressing towards clearance of the virus ${ }^{24}$ or have experienced clinical decline and hospitalization, further emphasizing the need for early intervention. As the clinical trial timelines typically represent an offset of several days from initial diagnosis, corresponding to day 10-14 of clinical illness, the actionable message remains unchanged - treat patients as early as possible to maximize the chance of altering the disease trajectory and promote recovery.

The COVID-19 pandemic poses logistical and medical challenges for the distribution of neutralizing mAbs. Up to $10 \%$ of initially asymptomatic, minimally symptomatic and mild infections progressed to severe disease including respiratory distress ${ }^{85}$. While approximately $78 \%$ of patients admitted to hospital have at least one documented co-morbidity ${ }^{86}$, there continue to be patients lacking any identified co-morbidity who subsequently become critically ill. Thus, the absence of co-morbidities does not completely eliminate the risk of severe disease and sequalae, and there is an urgent need for additional insight into a more personalized predictive algorithm to unlock as-yet-unidentified risk factors. Contrary to the discussion in the lay media, COVID-19 can potentially claim the lives of young adults in their prime, even in the absence of any known underlying risk factors. Given that persistently high SARS-CoV-2 viral loads may be associated with severe clinical outcomes ${ }^{87-89}$, it is possible that early reassessment of viral loads might help guide who among the 'lower-risk' population might be helped by neutralizing mAbs. RCT evidence indicates that the clinical value of neutralizing $\mathrm{mAb}$ therapy is more pronounced in individuals who are seronegative at diagnosis ${ }^{70}$. Collectively, measuring viral load and serology would allow strategic deployment for patients without otherwise identifiable risk factors while targeting early supply to the high-risk population. However, this strategy would be contingent on rapid turnaround of laboratory testing. Meanwhile, it also seems reasonable to use neutralizing mAbs early on during the disease for patients with well-identified risk factors for severe disease evolution ${ }^{90}$.

Another way to classify candidate patients for neutralizing mAbs would be to select patients who are expected to have poor antiviral responses (for example, elderly or immunocompromised patients) or to identify patients with poor T cell and/or B cell function via experimental techniques (such as by serology or flow cytometry). Regarding the latter, there is a lack of published evidence on humoral immune response dynamics and correlation with clinical outcomes. Furthermore, technical difficulties in stratifying patients on the basis of antibody production, lymphocyte function and/or viral load might pose a significant impediment to the timely identification of the most appropriate patients for neutralizing $\mathrm{mAb}$ therapy.

Finally, antiviral and antimicrobial therapies are traditionally plagued by their promoting escape variants, and sometimes combination therapy can mitigate this risk. As a first-generation approach for neutralizing mAbs, monotherapies have been developed and have been demonstrated to be efficacious, but it is expected that a greater number of combination therapies will follow. For example, in phase II/III trials involving patients with COVID-19, bamlanivimab and the bamlanivimab and etesevimab combination had similarly improved magnitudes and timings of symptom relief relative to the placebo ${ }^{25}$. However, to date, the bamlanivimab and etesevimab combination does not appear to lead to the emergence of drug-resistant variants of SARS-CoV-2. This is similar to what has been observed for the other authorized neutralizing $\mathrm{mAb}$ combination (casirivimab and imdevimab), as already described herein. At present, there remains a role for continued use of monotherapy while transitioning towards combination therapies so as to mitigate the selection pressure for viral escape as manufacturing capacity becomes available, to ensure longevity of the therapies and to reduce the potential rate of treatment failure.

\section{Summary and conclusions}

The sudden arrival and devastating spread of the COVID-19 pandemic has stimulated an accelerated programme of international research to identify effective ways to limit the spread of infection and to reduce the morbidity and mortality associated with COVID-19 $\left(\mathrm{REFS}^{91,92}\right)$. The first data are now emerging on vaccines designed to prevent disease ${ }^{93,94}$. In the context of active SARS-CoV-2 infection, clinical trials suggest that mortality in infected patients with hypoxia could be reduced with agents such as dexamethasone, baricitinib (in combination with remdesivir) and tocilizumab (data still under review) $)^{95-99}$. Furthermore, many trials have been conducted or are under way with various immune-modulating medications designed to limit the tissue damage associated with the later stages of COVID-19. However, to date there have been few unequivocal successes. Neutralizing mAbs, particularly in 
combination with other medications, are an attractive approach with potential utility in both prophylactic and treatment settings. Encouraging early clinical trial data support further investigation of neutralizing mAbs to determine the optimal dosing regimen. Unanswered questions regarding this novel therapeutic approach set a pressing research agenda; we need to establish which at-risk individuals would benefit most from prophylactic neutralizing $\mathrm{mAbs}$, the duration of protection offered by these $\mathrm{mAbs}$ and any potential impact of $\mathrm{mAb}$ therapy on subsequent vaccination. It will also be important to determine the optimum timing for administration of neutralizing $\mathrm{mAbs}$ on the basis of viral load, serology and other potential clinical factors.

Published online 19 April 2021
1. Renn, A., Fu, Y., Hu, X., Hall, M. D. \& Simeonov, A Fruitful neutralizing antibody pipeline brings hope to defeat SARS-Cov-2. Trends Pharmacol. Sci. 41, 815-829 (2020)

2. Shanmugaraj, B., Siriwattananon, K., Wangkanont, K. \& Phoolcharoen, W. Perspectives on monoclonal antibody therapy as potential therapeutic intervention for coronavirus disease-19 (COVID-19). Asian Pac. J. Allergy Immunol. 38, 10-18 (2020).

3. Regeneron Pharmaceuticals Inc. Fact sheet for health care providers: emergency use authorization (EUA) of casirivimab and imdevimab. Regeneron https:// www.regeneron.com/sites/default/files/treatmentcovid 19-eua-fact-sheet-for-hcp.pdf (2020).

4. US Food and Drug Administration. Fact sheet for health care providers emergency use authorization (EUA) of bamlanivimab. FDA https://www.fda.gov/ media/143603/download (2020).

5. US Food and Drug Administration. Fact sheet for health care providers emergency use authorization (EUA) of bamlanivimab and etesevimab. FDA https:// www.fda.gov/media/145802/download (2021).

6. Regeneron Pharmaceuticals Inc. Regeneron reports positive interim data with REGEN-COV ${ }^{\text {th }}$ antibody cocktail used as passive vaccine to prevent COVID-19. Regeneron https://newsroom.regeneron.com/newsreleases/news-release-details/regeneron-reportspositive-interim-data-regen-covtm-antibody (2021).

7. Eli Lilly and Company. Lilly's neutralizing antibody bamlanivimab (LY-CoV555) prevented COVID-19 at nursing homes in the BLAZE-2 trial, reducing risk by up to 80 percent for residents. Eli Lilly and Company https://investor.lilly.com/news-releases/news-releasedetails/lillys-neutralizing-antibody-bamlanivimably-cov555-prevented (2021).

8. Llewelyn, M. B., Hawkins, R. E. \& Russell, S. J. Discovery of antibodies. BMJ 305, 1269-1272 (1992).

9. Behring, E. A. \& Kitasato, S. Ueber das Zustandekommen der Diphtherie-immunitat und der Tetanus-immunitat bei Thieren. Dtsch. Med. Wochenschr. 28, 1321-1332 (1890)

10. Kaufmann, S. H. Immunology's foundation: the 100-year anniversary of the Nobel Prize to Paul Ehrlich and Elie Metchnikoff. Nat. Immunol. 9, 705-712 (2008)

11. Hung, I. F. et al. Convalescent plasma treatment reduced mortality in patients with severe pandemic influenza A (H1 N1) 2009 virus infection. Clin. Infect. Dis. 52, 447-456 (2011).

12. Mair-Jenkins, J. et al. The effectiveness of convalescent plasma and hyperimmune immunoglobulin for the treatment of severe acute respiratory infections of viral etiology: a systematic review and exploratory meta-analysis. J. Infect. Dis. 211, 80-90 (2015).

13. Groothuis, J. R. et al. Prophylactic administration of respiratory syncytial virus immune globulin to high-risk infants and young children. N. Engl. J. Med. 329, 1524-1530 (1993)

14. Sahr, F. et al. Evaluation of convalescent whole blood for treating Ebola virus disease in Freetown, Sierra Leone. J. Infect. 74, 302-309 (2017).

15. Cheng, Y. et al. Use of convalescent plasma therapy in SARS patients in Hong Kong. Eur. J. Clin. Microbiol. Infect. Dis. 24, 44-46 (2005).

16. Yeh, K. M. et al. Experience of using convalescent plasma for severe acute respiratory syndrome among healthcare workers in a Taiwan hospital. J. Antimicrob. Chemother. 56, 919-922 (2005).

17. Ko, J. H. et al. Challenges of convalescent plasma infusion therapy in Middle East respiratory coronavirus infection: a single centre experience. Antivir. Ther. 23 , 617-622 (2018)

18. Joyner, M. J. et al. Effect of convalescent plasma on mortality among hospitalized patients with COVID-19 initial three-month experience. Preprint at medRxiv https://doi.org/10.1101/2020.08.12.20169359 (2020).
19. Zhang, J. S. et al. A serological survey on neutralizing antibody titer of SARS convalescent sera. J. Med. Virol. 77, 147-150 (2005)

20. Iwasaki, A. $\&$ Yang, Y. The potential danger of suboptimal antibody responses in COVID-19. Nat. Rev. Immunol. 20, 339-341 (2020).

21. Yip, M. et al. Antibody-dependent enhancement of SARS coronavirus infection and its role in the pathogenesis of SARS. Hong. Kong Med. J. 22, 25-31 (2016).

22. Lee, W. S., Wheatley, A. K., Kent, S. J. \& DeKosky, B. J. Antibody-dependent enhancement and SARS-CoV-2 vaccines and therapies. Nat. Microbiol. 5, 1185-1191 (2020).

23. Wang, Q. et al. Immunodominant SARS coronavirus epitopes in humans elicited both enhancing and neutralizing effects on infection in non-human primates. ACS infect. Dis. 2, 361-376 (2016).

24. Chen, P. et al. SARS-CoV-2 neutralizing antibody LYCoV555 in outpatients with Covid-19. N. Engl. J. Med https://doi.org/10.1056/NEJMoa2029849 (2020).

25. Gottlieb, R. L. et al. Effect of bamlanivimab as monotherapy or in combination with etesevimab on viral load in patients with mild to moderate COVID-19. a randomized clinical trial. JAMA https://doi.org/ 10.1001/jama.2021.0202 (2021).

26. US Food and Drug Administration. Recommendations for investigational COVID-19 convalescent plasma. FDA https://www.fda.gov/vaccines-blood-biologics/ investigational-new-drug-ind-or-device-exemption-ideprocess-cber/recommendations-investigationalcovid-19-convalescent-plasma (2020).

27. US Food and Drug Administration. Convalescent plasma COVID-19 letter of authorization. FDA https:// www.fda.gov/media/141477/download (2020).

28. Davis, C. W. et al. Longitudinal analysis of the human B cell response to Ebola virus infection. Cell 177, 1566-1582 (2019).

29. The Antibody Society. Therapeutic monoclonal antibodies approved or in review in the EU or US The Antibody Society https://www.antibodysociety.org/ resources/approved-antibodies/ (2020).

30. Pecetta, S., Finco, O. \& Seubert, A. Quantum leap of monoclonal antibody (mAb) discovery and developmen in the COVID-19 era. Semin. Immunol. 50, 101427 (2020).

31. The IMpact-RSV Study Group. Palivizumab, a humanized respiratory syncytial virus monoclonal antibody, reduces hospitalization from respiratory syncytial virus infection in high-risk infants. Pediatrics 102, 531-537 (1998).

32. Feltes, T. F. et al. Palivizumab prophylaxis reduces hospitalization due to respiratory syncytial virus in young children with hemodynamically significant congenital heart disease. J. Pediatr. 143, 532-540 (2003).

33. Elawar, F. et al. Pharmacological targets and emerging treatments for respiratory syncytial virus bronchiolitis. Pharmacol. Ther. https://doi.org/10.1016/ j.pharmthera.2020. (2020).

34. Helmink, B. J., Ragsdale, C. E., Peterson, E. J. \& Merkel, K. G. Comparison of intravenous palivizumab and standard of care for treatment of respiratory syncytial virus infection in mechanically ventilated pediatric patients. J. Pediatr. Pharmacol. Ther. 21, 146-154 (2016).

35. Sáez-Llorens, X. et al. Safety and pharmacokinetics of palivizumab therapy in children hospitalized with respiratory syncytial virus infection. Pediatr. Infect. Dis. J. 23, 707-712 (2004).

36. Malley, R. et al. Reduction of respiratory syncytial virus (RSV) in tracheal aspirates in intubated infants by use of humanized monoclonal antibody to RSV F protein. J. Infect. Dis. 178, 1555-1561 (1998).

37. Soto, J. A., Gálvez, N. M. S., Pacheco, G. A., Bueno, S. M. \& Kalergis, A. M. Antibody development for preventing the human respiratory syncytial virus pathology. Mol. Med. https://doi.org/10.1186/ s10020-020-00162-6 (2020).

38. Mulangu, S. et al. A randomized, controlled trial of Ebola virus disease therapeutics. N. Engl. J. Med. 381, 2293-2303 (2019).

39. Crowe, J. E. Jr., Firestone, C. Y. \& Murphy, B. R. Passively acquired antibodies suppress humoral but not cell-mediated immunity in mice immunized with live attenuated respiratory syncytial virus vaccines. J. Immunol. 167, 3910-3918 (2001).

40. Crowe, J. E. Jr. et al. Cold-passaged, temperature sensitive mutants of human respiratory syncytial virus (RSV) are highly attenuated, immunogenic, and protective in seronegative chimpanzees, even when RSV antibodies are infused shortly before immunization. Vaccine 13, 847-855 (1995).

41. Liu, Z. et al. Identification of SARS-CoV-2 spike mutations that attenuate monoclonal and serum antibody neutralization. Cell Host Microbe https:// doi.org/10.1016/j.chom.2021.01.014 (2021).

42. Wang, P. et al. Increased resistance of SARS-CoV-2 variants B. 1.351 and B. 1.1. 7 to antibody neutralization. Preprint at bioRxiv https://doi.org/ 10.1101/2021.01.25.428137 (2021).

43. Bar-On, Y. et al. Safety and antiviral activity of combination HIV-1 broadly neutralizing antibodies in viremic individuals. Nat. Med. 24, 1701-1707 (2018).

44. Mendoza, P. et al. Combination therapy with antiHIV- 1 antibodies maintains viral suppression. Nature 561, 479-484 (2018).

45. Caskey, M. et al. Antibody 10-1074 suppresses viremia in HIV-1-infected individuals. Nat. Med. 23, 185-191 (2017).

46. Scheid, J. F. et al. HIV-1 antibody 3BNC117 suppresses viral rebound in humans during treatment interruption. Nature 535, 556-560 (2016)

47. Ho, M. S. et al. Neutralizing antibody response and SARS severity. Emerg. Infect. Dis. 11, 1730-1737 (2005).

48. Zhang, L. et al. Antibody responses against SARS coronavirus are correlated with disease outcome of infected individuals. J. Med. Virol. 78, 1-8 (2006).

49. Wu, F. et al. Neutralizing antibody responses to SARS-CoV-2 in a COVID-19 recovered patient cohort and their implications. Preprint at medRxiv https:// doi.org/10.1101/2020.03.30.20047365 (2020).

50. Long, Q. X. et al. Antibody responses to SARS-CoV-2 in patients with COVID-19. Nat. Med. 26, 845-848 (2020).

51. Li, K. et al. Dynamic changes in anti-SARS-CoV-2 antibodies during SARS-CoV-2 infection and recovery from COVID-19. Nat Commun 11, 6044 (2020).

52. Ouyang, J. et al. Convalescent plasma: the relay baton in the race for coronavirus disease 2019 treatment. Front. Immunol. 11, 570063 (2020).

53. Shen, C. et al. Treatment of 5 critically ill patients with COVID-19 with convalescent plasma. JAMA 323, 1582-1589 (2020).

54. Li, L. et al. Effect of convalescent plasma therapy on time to clinical improvement in patients with severe and life-threatening COVID-19: a randomized clinical trial. JAMA 324, 460-470 (2020).

55. Simonovich, V. A. et al. A randomized trial of convalescent plasma in COVID-19 severe pneumonia. N. Engl. J. Med. https://doi.org/10.1056/ NEJMoa2031304 (2020).

56. Agarwal, A. et al. Convalescent plasma in the management of moderate covid-19 in adults in India: open label phase II multicentre randomised controlled trial (PLACID trial). BMJ 371, m3939 (2020).

57. Sandercock, P. RECOVERY trial DMC report. University of Edinburgh https://www. recoverytrial.net/ files/recovery-dmc-letter-14-january-2021.pdf (2021).

58. RECOVERY Trial Chief Investigators. RECOVERY trial closes recruitment to convalescent plasma treatment for patients hospitalised with COVID-19. Nuffield 
Department of Population Health https://www. recoverytrial.net/news/statement-from-the-recoverytrial-chief-investigators-15-january-2021-recovery-trialcloses-recruitment-to-convalescent-plasma-treatmentfor-patients-hospitalised-with-covid-19 (2021).

59. Du, L. et al. The spike protein of SARS-CoV-a target for vaccine and therapeutic development. Nat. Rev. Microbiol. 7, 226-236 (2009).

60. Lu, R. et al. Genomic characterisation and epidemiology of 2019 novel coronavirus: implications for virus origins and receptor binding. Lancet 395, 565-574 (2020).

61. Wan, Y., Shang, J., Graham, R., Baric, R. S. \& Li, F. Receptor recognition by the novel coronavirus from Wuhan: an analysis based on decade-long structural studies of SARS coronavirus. J. Virol. https://doi.org/ 10.1128/JVI.00127-20 (2020)

62. Hoffmann, M., Kleine-Weber, H. \& Pöhlmann, S A multibasic cleavage site in the spike protein of SARS-CoV-2 is essential for infection of human lung cells. Mol. Cell 78, 779-784.e775 (2020).

63. Klasse, P. J. \& Moore, J. P. Antibodies to SARS-CoV-2 and their potential for therapeutic passive immunization. eLife 9, e57877 (2020).

64. Chen, X. et al. Human monoclonal antibodies block the binding of SARS-CoV-2 spike protein to angiotensin converting enzyme 2 receptor. Cell Mol. Immunol. 17, 647-649 (2020).

65. Wu, Y. et al. A noncompeting pair of human neutralizing antibodies block COVID-19 virus binding to its receptor ACE2. Science 368, 1274-1278 (2020).

66. Jones, B. E. et al. The neutralizing antibody LY-CoV555, protects against SARS-CoV-2 infection in non-human primates. Sci. Trans/ Med. https://doi.org/ 10.1126/scitranslmed.abf1906 (2021).

67. Baum, A. et al. Antibody cocktail to SARS-CoV-2 spike protein prevents rapid mutational escape seen with individual antibodies. Science 369, 1014-1018 (2020).

68. Hansen, J. et al. Studies in humanized mice and convalescent humans yield a SARS-CoV-2 antibody cocktail. Science 369, 1010-1014 (2020).

69. Baum, A. et al. REGN-COV2 antibodies prevent and treat SARS-CoV-2 infection in rhesus macaques and hamsters. Science 370, 1110-1115 (2020).

70. Regeneron Pharmaceuticals Inc. Regeneron's COVID-19 outpatient trial prospectively demonstrates that REGN-COV2 antibody cocktail significantly reduced virus levels and need for further medical attention. Regeneron https://investor.regeneron.com/newsreleases/news-release-details/regenerons-covid-19outpatient-trial-prospectively-demonstrates (2020).

71. Regeneron Pharmaceuticals Inc. Regeneron's casirivimab and imdevimab antibody cocktail for COVID-19 is first combination therapy to receive FDA emergency use authorization. Regeneron https:// investor.regeneron.com/news-releases/news-releasedetails/regenerons-regen-cov2-first-antibody-cocktailcovid-19-receive (2020).

72. Shi, R. et al. A human neutralizing antibody targets the receptor-binding site of SARS-CoV-2. Nature $\mathbf{5 8 4}$ 120-124 (2020)

73. Eli Lilly and Company. New data show treatment with Lilly's neutralizing antibodies bamlanivimab (LY-CoV555) and etesevimab (LY-CoV016) together reduced risk of COVID-19 hospitalizations and death by 70 percent. Eli Lilly and Company https://investor lilly.com/news-releases/news-release-details/new-datashow-treatment-lillys-neutralizing-antibodies (2021).

74. Regeneron Pharmaceuticals Inc. REGN-COV2 independent data monitoring committee recommends holding enrollment in hospitalized patients with high oxygen requirements and continuing enrollment in patients with low or no oxygen requirements. Regeneron https://investor.regeneron.com/newsreleases/news-release-details/regn-cov2-independentdata-monitoring-committee-recommends (2020).

75. Regeneron Pharmaceuticals Inc. RECOVERY Trial Data Monitoring Committee recommends continuing evaluation of REGN-COV2 in all hospitalized patients. Regeneron https://investor.regeneron.com/newsreleases/news-release-details/recovery-trial-datamonitoring-committee-recommends-continuing (2020).

76. Regeneron Pharmaceuticals Inc. Regeneron announces encouraging initial data from COVID-19 antibody cocktail trial in hospitalized patients on low-flow oxygen. Regeneron https://investor.regeneron.com/ news-releases/news-release-details/regeneronannounces-encouraging-initial-data-covid-19-antibody (2020).

77. ACTIV-3/TICO LY-CoV555 Study Group. A neutralizing monoclonal antibody for hospitalized patients with COVID-19. N. Engl. J. Med. https://doi.org/10.1056/ NEJMoa2033130 (2020)

78. Weinreich, D. M. et al. REGN-COV2, a neutralizing antibody cocktail, in outpatients with COVID-19. N. Engl. J. Med. 34, 238-251 (2020).

79. Greaney, A. J. et al. Complete mapping of mutations to the SARS-CoV-2 spike receptor-binding domain that escape antibody recognition. Cell Host Microbe 29 44-57 (2021)

80. Thomson, E. C. et al. The circulating SARS-CoV-2 spike variant $\mathrm{N} 439 \mathrm{~K}$ maintains fitness while evading antibody-mediated immunity. Cell 184, 1171-1187 (2021).

81. Mbala-Kingebeni, P. et al. Medical countermeasures during the 2018 Ebola virus disease outbreak in th North Kivu and Ituri provinces of the Democratic Republic of the Congo: a rapid genomic assessment. Lancet Infect. Dis. 19, 648-657 (2019).

82. GISAID Initiative. Relative variant genome frequency per region. GISAID https://www.gisaid.org/hcov19variants/ (2021).

83. Centers for Disease Control and Prevention. New variants of the virus that causes COVID-19. CDC https://www.cdc.gov/coronavirus/2019-ncov/ transmission/variant.html (2021)

84. Zhang, G. et al. The basis of a more contagious 501Y. V1 variant of SARS-COV-2. Preprint at bioRxiv https://doi.org/10.1101/2021.02.02.428884 (2021).

85. Tabata, S. et al. Clinical characteristics of COVID-19 in 104 people with SARS-CoV-2 infection on the Diamond Princess cruise ship: a retrospective analysis. Lancet Infect. Dis. 20, 1043-1050 (2020).

86. Docherty, A. B. et al. Features of 20133 UK patients in hospital with covid-19 using the ISARIC WHO Clinical Characterisation Protocol: prospective observational cohort study. BMJ 369, m 1985 (2020)

87. Chen, J. et al. Clinical progression of patients with COVID-19 in Shanghai, China. J. Infect. 80, e1-e6 (2020).

88. Liu, Y. et al. Viral dynamics in mild and severe cases of COVID-19. Lancet Infect. Dis. 20, 656-657 (2020).

89. Westblade, L. F. et al. SARS-CoV-2 viral load predicts mortality in patients with and without cancer who are hospitalized with COVID-19. Cancer Cell 38, 661-67 (2020).

90. Center for Disease Control and Prevention. People at increased risk and other people who need to take extra precautions. CDC https://www.cdc.gov/ coronavirus/2019-ncov/need-extra-precautions/index. html?CDC_AA_refVal=https $\% 3 \mathrm{~A} \% 2 \mathrm{~F} \% 2 \mathrm{Fwww} . \mathrm{cdc}$. gov\%2Fcoronavirus\%2F2019-ncov\%2Fneed-extraprecautions \%2Fpeople-at-increased-risk.html (2021).
91. Wang, C., Horby, P. W., Hayden, F. G. \& Gao, G. F. A novel coronavirus outbreak of global health concern. Lancet 395, 470-473 (2020)

92. Zhu, N. et al. A novel coronavirus from patients with pneumonia in China, 2019. N. Engl. J. Med. 382 727-733 (2020).

93. Dai, L. \& Gao, G. F. Viral targets for vaccines against COVID-19. Nat. Rev. Immunol. 21, 73-82 (2020).

94. Krammer, F. SARS-CoV-2 vaccines in development. Nature 586, 516-527 (2020)

95. Horby, P. et al. Dexamethasone in hospitalized patients with Covid-19 - preliminary report. N. Engl. J. Med. https://doi.org/10.1056/NEJMoa2021436 (2020).

96. Kalil, A. C. et al. Baricitinib plus remdesivir for hospitalized adults with Covid-19. N. Engl. J. Med. https://doi.org/10.1056/NEJMoa2031994 (2020).

97. US Food and Drug Administration. Fact sheet for healthcare providers emergency use authorization (EUA) of baricitinib. FDA https://www.fda.gov/ media/143823/download (2020)

98. The REMAP-CAP Investigators et al. Interleukin-6 receptor antagonists in critically ill patients with Covid 19-preliminary report. Preprint at medRxiv https://doi.org/10.1101/2021.01.07.21249390 (2021).

99. RECOVERY Collaborative Group et al. Tocilizumab in patients admitted to hospital with COVID-19 (RECOVERY): preliminary results of a randomised, controlled, open-label, platform trial. Preprint at medRxiv https://doi.org/10.1101/2021.02.11. 21249258 (2021).

\section{Acknowledgements}

This work was supported by Eli Lilly and Company. C. J. Antalis and H. Green (Eli Lilly and Company) provided editorial assistance.

\section{Author contributions}

The authors contributed equally to all aspects of the article.

\section{Competing interests}

P.C.T. has received research grants, consultation fees and/or speaking fees from AbbVie, Biogen, Bristol-Myers Squibb, Celgene, Celltrion, Fresenius, Galapagos, Gilead, GlaxoSmithKline, Janssen, Eli Lilly and Company, Sanofi, Nordic Pharma, Pfizer, Roche and UCB. A.C.A., I.d.I.T. and M.M.H. are employees and shareholders of Eli Lilly and Company. K.W. has received research grants from BristolMyers Squibb and Pfizer and consulting fees from AbbVie, AstraZeneca, Bristol-Myers Squibb, Eli Lilly and Company, Galapagos, GlaxoSmithKline, Gilead, Novartis, Pfizer, Regeneron, Roche, Sanofi and UCB. R.L.G. reports nonfinancial support from Gilead Sciences Inc. and personal fees from Gilead Sciences Inc. outside the submitted work.

\section{Peer review information}

Nature Reviews Immunology thanks the anonymous reviewers for their contribution to the peer review of this work.

\section{Publisher's note}

Springer Nature remains neutral with regard to jurisdictional claims in published maps and institutional affiliations.

\section{RELATED LINKS}

ClinicalTrials.gov: https://clinicaltrials.gov

COVID-19 Biologics Tracker: https://www.antibodysociety. org/covid-19-biologics-tracker/

GISAID COVID-19 variant tracker: https://www.gisaid.org/

(c) The Author(s), under exclusive licence to Springer Nature Limited. 2021 